\begin{tabular}{|c|c|}
\hline Title & $\begin{array}{l}\text { Suppression of metal-insulator transition at high pressure and pressure induced magnetic ordering in pyrochlore oxide } \\
\text { Nd2l } 207\end{array}$ \\
\hline Author(s) & $\begin{array}{l}\text { Sakata, Masafumi; Kagay ama, Tomoko; Shimizu, Katsuya; Matsuhira, Kazuyuki; Takagi, Seishi; Wakeshima, Makoto; } \\
\text { Hinatsu, Y ukio }\end{array}$ \\
\hline Citation & $\begin{array}{l}\text { Physical Review B, 83(4), } 041102 \\
\text { https://doi.org/10.1103PhysRevB.83.041102 }\end{array}$ \\
\hline Issue Date & 2011-01-01 \\
\hline Doc URL & http:/hdl. handle.net/2115/44975 \\
\hline Rights & (-2011 A merican Physical Society \\
\hline Type & article \\
\hline File Information & PRB83-4_041102.pdf \\
\hline
\end{tabular}

Instructions for use 


\title{
Suppression of metal-insulator transition at high pressure and pressure-induced magnetic ordering in pyrochlore oxide $\mathrm{Nd}_{2} \mathrm{Ir}_{2} \mathrm{O}_{7}$
}

\author{
Masafumi Sakata, ${ }^{*}$ Tomoko Kagayama, and Katsuya Shimizu \\ KYOKUGEN, Osaka University, Machikaneyama-cho, Toyonaka, Osaka 560-8531, Japan
}

Kazuyuki Matsuhira and Seishi Takagi

Faculty of Engineering, Kyusyu Institute of Technology, Kitakyusyu 804-8550, Japan

Makoto Wakeshima and Yukio Hinatsu

Division of Chemistry, Graduate School of Science, Hokkaido University, Sapporo 060-0810, Japan

(Received 12 February 2010; published 10 January 2011)

\begin{abstract}
We report the pressure dependence of electrical resistance $R(T)$ of a frustrated pyrochlore oxide $\mathrm{Nd}_{2} \mathrm{Ir}_{2} \mathrm{O}_{7}$, which shows a second-order metal-insulator transition (MIT) at $36 \mathrm{~K}$. This MIT is monotonically suppressed by the application of pressure. The insulating phase disappears at around $10 \mathrm{GPa}$. Then, in the pressure-induced metallic state above $10 \mathrm{GPa}$, a new pressure-induced phase transition emerges at around $3 \mathrm{~K}$, which is characterized by a resistance drop. The new phase transition is due to a ferromagnetic (FM) ordering, which is suggested to be an ordered spin ice (two-in two-out) state of the Nd moment via the RKKY interaction. We succeeded in observing the phase competition between the MIT and the FM ordering by the application of high pressure.
\end{abstract}

DOI: 10.1103/PhysRevB.83.041102

PACS number(s): 71.30.+h, 72.15.-v, 75.47.-m, 75.30.Mb

Geometrical frustration in a magnetic material can lead to novel phenomena such as a macroscopic degeneracy in the ground state with no long-range ordering (LRO). Pyrochlore oxides $A_{2} B_{2} \mathrm{O}_{7}$ have attracted great interest because their structure, ${ }^{1}$ which includes corner-shared tetrahedra whose vertices are occupied by spins, may show a strong geometrical frustration such as spin ice ${ }^{2,3}$ and spin liquid states. ${ }^{4}$ Furthermore, in metallic pyrochlore oxides, a frustration originating from the pyrochlore lattice might also lead to novel types of electronic properties. In fact, $4 d$ and $5 d$ transition-metal pyrochlore oxides have been reported to show various transport properties such as superconductivity in $\mathrm{Cd}_{2} \mathrm{Re}_{2} \mathrm{O}_{7}{ }^{5}$ and $A \mathrm{Os}_{2} \mathrm{O}_{6}$ (where $A=\mathrm{K}, \mathrm{Rb}$, and $\mathrm{Cs}$ ), ${ }^{6-8}$ the unconventional anomalous Hall effect in $\mathrm{Nd}_{2} \mathrm{Mo}_{2} \mathrm{O}_{7}{ }^{9}$ and $\mathrm{Pr}_{2} \mathrm{Ir}_{2} \mathrm{O}_{7},{ }^{10}$ and metal-insulator transition (MIT) in $\mathrm{Cd}_{2} \mathrm{Os}_{2} \mathrm{O}_{7},{ }^{11,12} \mathrm{Tl}_{2} \mathrm{Ru}_{2} \mathrm{O}_{7},{ }^{13,14}$ and $\mathrm{Hg}_{2} \mathrm{Ru}_{2} \mathrm{O}_{7} .{ }^{15}$

The strongly frustrated state can be relieved by the application of a magnetic field and pressure. Applying a magnetic field is the easiest method to lower the symmetry of the system. Actually, the application of a magnetic field in the [111] direction to the spin ice state induces a Kagomé ice state, where the macroscopic degeneracy is partially reduced. ${ }^{16}$ On the other hand, applying pressure is an effective method to drive more dramatic change in various physical parameters such as magnetic interactions, bandwidth, and electron correlations. In fact, the application of high pressure to the spin liquid $\mathrm{Tb}_{2} \mathrm{Ti}_{2} \mathrm{O}_{7}$ induces LRO. ${ }^{17}$ Furthermore, the application of pressure on the MIT in pyrochlore oxides stabilizes it to a metallic state; the negative pressure dependence of the transition temperature $\left(T_{\mathrm{MI}}\right)$ has been observed in $\mathrm{Cd}_{2} \mathrm{Os}_{2} \mathrm{O}_{7}$ $\left(T_{\mathrm{MI}}=226 \mathrm{~K}\right)^{12}$ and $\mathrm{Hg}_{2} \mathrm{Ru}_{2} \mathrm{O}_{7}\left(T_{\mathrm{MI}}=107 \mathrm{~K}\right) .{ }^{18}$ In particular, for $\mathrm{Hg}_{2} \mathrm{Ru}_{2} \mathrm{O}_{7}$, a Fermi liquid with an enhanced mass is realized above $6.5 \mathrm{GPa} .{ }^{18}$ Thus, the application of pressure to the strongly frustrated state can lead to a novel phase.

A recent study on pyrochlore iridates $L_{2} \mathrm{Ir}_{2} \mathrm{O}_{7}$ has revealed the occurrence of MITs for $L n=\mathrm{Nd}, \mathrm{Sm}$, and Eu at $T_{\mathrm{MI}}$ of 36,
117 , and $120 \mathrm{~K}$, respectively. ${ }^{19}$ These MITs are second-order transitions and involve a magnetic anomaly caused by $5 d$ electrons from $\mathrm{Ir}^{4+}$. It should be noted that $T_{\mathrm{MI}}$ increases with decreasing the ionic radius of $\mathrm{Ln}^{3+}$. Furthermore, the electrical conductivity of the iridates also depends on this ionic radius. With decreasing ionic radius of $\mathrm{Ln}^{3+}$, the Ir-O-Ir bond angle decreases, and, consequently, the $t_{2 g}$ bandwidth becomes narrower. The metallic conductivity of $\operatorname{Pr}_{2} \mathrm{Ir}_{2} \mathrm{O}_{7}$ is retained at least down to $0.3 \mathrm{~K}$ without the occurrence of the MIT. ${ }^{10}$ On changing $L n$ from Pr to Eu, the electrical conductivity changes from metallic to semimetallic. Further, $\mathrm{Y}_{2} \mathrm{Ir}_{2} \mathrm{O}_{7}$ with a smaller ionic radius acts as a Mott insulator because of strong electron correlations. $^{20,21}$ However, the origin of continuous MIT in $\mathrm{Ln}_{2} \mathrm{Ir}_{2} \mathrm{O}_{7}$ is still unclear. Therefore, experimental and theoretical studies for determining the origin are currently in progress.

The application of external pressure to $\mathrm{Ln}_{2} \mathrm{Ir}_{2} \mathrm{O}_{7}$ perturbs its crystal structure directly. Therefore, we can expect to control the MIT in $\mathrm{Ln}_{2} \mathrm{Ir}_{2} \mathrm{O}_{7}$ by the application of high pressure, since this pressure application changes the unit cell size, which in turn changes $T_{\mathrm{MI}}$. Because of this ionic radius dependence of $T_{\mathrm{MI}}$, we speculate that the application of pressure may lead to an increase in $T_{\mathrm{MI}}$. However, this speculated pressure effect is contradictory to the negative pressure dependence of $T_{\mathrm{MI}}$ observed in $\mathrm{Cd}_{2} \mathrm{Os}_{2} \mathrm{O}_{7}{ }^{12}$ and $\mathrm{Hg}_{2} \mathrm{Ru}_{2} \mathrm{O}_{7} .{ }^{18}$ Therefore, the effect of pressure application on the MIT in $L n_{2} \mathrm{Ir}_{2} \mathrm{O}_{7}$ is nontrivial. As $\mathrm{Nd}_{2} \mathrm{Ir}_{2} \mathrm{O}_{7}$ is nearly located in the ionic radius boundary for MITs in $L n_{2} \mathrm{Ir}_{2} \mathrm{O}_{7}$, a dramatic change of MIT is expected. Furthermore, it is speculated that the suppression of the MIT to low temperature by the application of pressure would result in the formation of new phases.

In this study, single crystals of $\mathrm{Nd}_{2} \mathrm{Ir}_{2} \mathrm{O}_{7}$ (with a volume of approximately $0.01 \mathrm{~mm}^{3}$ ) were grown using a KF-flux method reported in Ref. 22. Pressure was generated using a diamond anvil cell. Sodium chloride was used as a pressure medium; as a result the applied pressure was quasihydrostatic. Pressure 

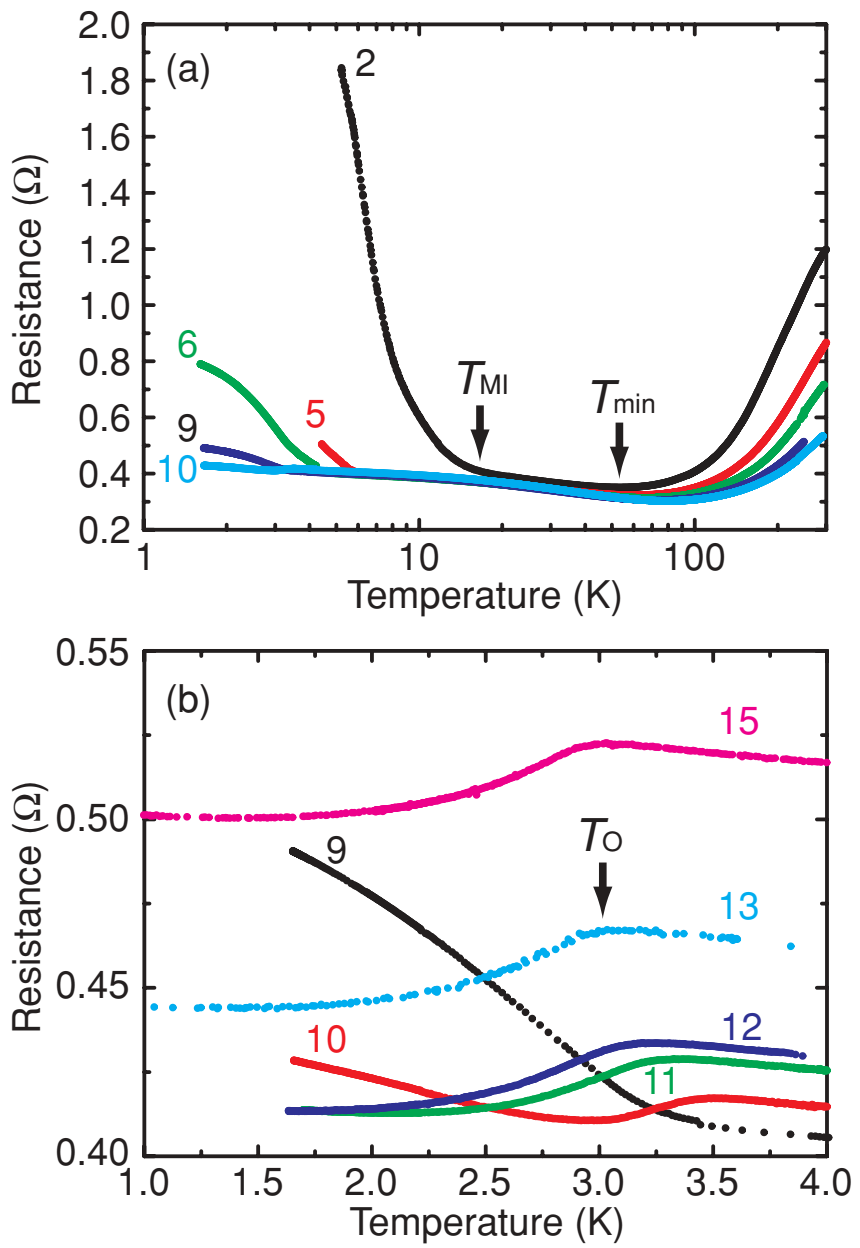

FIG. 1. (Color online) $R(T)$ at (a) pressure up to $11 \mathrm{GPa}$ and (b) pressure greater than $8 \mathrm{GPa}$. The numerals on the $R(T)$ curves denote the value of pressure.

was determined by the standard ruby fluorescence method at room temperature; we ensured that the pressure change at low temperature was smaller than $0.3 \mathrm{GPa}$. A four-probe method was employed for resistance measurement without determination of the direction in the single crystal. The resistance was measured by a dc method with excitation currents of 100 and $10 \mu \mathrm{A}$, in which the measured temperature dependence of resistance $[R(T)]$ show no difference between the two current values. We show $R(T)$ with $100-\mu \mathrm{A}$ excitation current in this Rapid Communication. At $2 \mathrm{GPa}$, the lowest pressure in this measurement, the resistivity at room temperature was roughly estimated to be about $4 \mathrm{~m} \Omega \mathrm{cm}$ by using the size of the sample under ambient pressure.

Figure 1 shows $R(T)$ at an applied pressure greater than $2 \mathrm{GPa}$. As $R(T)$ at around room temperature gradually decreases with increasing pressure, the system becomes metallic. $T_{\mathrm{MI}}$ and $R(T)$ in the insulating phase also decrease with increasing pressure. In the insulating phase, the tiny slope change of $R(T)$ was observed up to $9 \mathrm{GPa}$. This probably indicates that the band gap is incompletely opened under the pressure. From these results, we could confirm that the insulating phase is suppressed by the application of pressure. The MIT has been found to be continuous at ambient pressure ${ }^{19}$ and at pressures less than $9 \mathrm{GPa}$ based on the absence

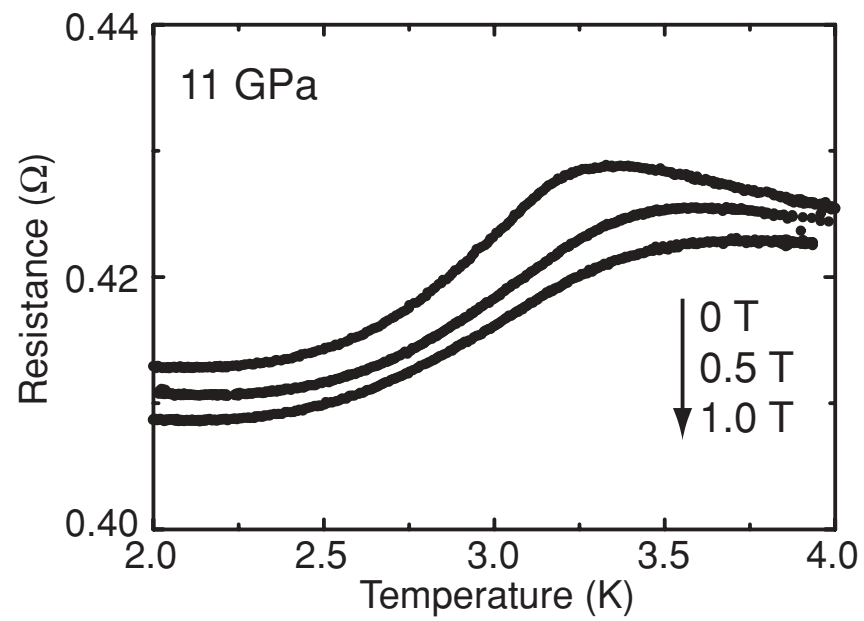

FIG. 2. $R(T)$ at $11 \mathrm{GPa}$ under several magnetic fields.

of discontinuities and thermal hysteresis of the MIT. Once the pressure increases to approximately $11 \mathrm{GPa}$, no MIT is observed in the measured temperature region.

In a previous study, a minimum in resistivity of a polycrystalline sample at ambient pressure was not observed. ${ }^{19}$ Here, the minimum in $R(T)$ occurs in the metallic phase, at a temperature denoted as $T_{\min }$ in Fig. 1(a). This minimum became observable by the suppression of the MIT under pressure. With increasing pressure, $T_{\min }$ shifts toward high temperature up to $13 \mathrm{GPa}$. Above $13 \mathrm{GPa}, T_{\min }$ decreases with increasing pressure. It should be noted that a minimum in $R(T)$ has also been observed in similar pyrochlore iridate $\mathrm{Pr}_{2} \mathrm{Ir}_{2} \mathrm{O}_{7} .{ }^{23}$ The present result suggests that the resistance minimum is a feature common to $\mathrm{Ln}_{2} \mathrm{Ir}_{2} \mathrm{O}_{7}$ in the metallic state. Therefore, there is a possibility that the resistance minimum of $\mathrm{Nd}_{2} \mathrm{Ir}_{2} \mathrm{O}_{7}$ is caused by the Kondo effect, as is the case with $\mathrm{Pr}_{2} \mathrm{Ir}_{2} \mathrm{O}_{7}$. However, we do not have additional information that would elucidate the exact reason for the resistance minimum. Therefore, a further discussion of $T_{\min }$ is beyond the scope of this Rapid Communication.

As shown in Fig. 1(b), at a pressure of $10 \mathrm{GPa}, R(T)$ drops at around $3.5 \mathrm{~K}$ and increases slightly at $3 \mathrm{~K}$. This slight increase corresponds to the MIT observed at lower pressures. With increasing pressure, the MIT is suppressed completely but the drop in $R(T)$ remained up to around $15 \mathrm{GPa}$. This drop indicates a new pressure-induced phase transition. Figure 2 shows $R(T)$ at $11 \mathrm{GPa}$ under several magnetic fields. At a pressure of $11 \mathrm{GPa}$, a drop of $4 \%$ in $R(T)$ at $0 \mathrm{~T}$ is observed for $T_{\mathrm{O}}=$ $3.2 \mathrm{~K}$, where $T_{\mathrm{O}}$ is the onset temperature of the resistance drop. With increasing magnetic field, the drop in $R(T)$ broadens and $R(T)$ starts to decrease at higher temperature. Furthermore, the magnetoresistance is negative. The magnetic field dependence indicates that the drop in $R(T)$ is caused by a ferromagnetic (FM) transition.

Next, we will discuss the pressure-induced FM ordering in a pyrochlore lattice in the metallic state. As the origin of the FM ordering, there are three possibilities: ordering of the Ir moment, the $\mathrm{Nd}$ moment, or both $\mathrm{Ir}$ and $\mathrm{Nd}$ moments. First, it is important to consider the degree of freedom of the localized Nd moment. The crystalline electric field with $D_{3 d}$ symmetry splits the ground state $J=9 / 2$ multiplet in $\mathrm{Nd}^{3+}$ into 
five Kramers doublets. In this case, the Kramers ground-state doublet has a magnetic degree of freedom. The ground-state entropy of $R \ln 2$ has to be relieved down to $0 \mathrm{~K}$. It is known that the $\mathrm{Nd}$ moment in pyrochlore oxide, $\mathrm{Nd}_{2} \mathrm{Mo}_{2} \mathrm{O}_{7}$, has a local $\langle 111\rangle$ Ising anisotropy. ${ }^{10}$ Our preliminary magnetization measurement of $\mathrm{Nd}_{2} \mathrm{Ir}_{2} \mathrm{O}_{7}$ indicates that the $\mathrm{Nd}$ moment also has a local $\langle 111\rangle$ Ising anisotropy. ${ }^{24}$

A local $\langle 111\rangle$ Ising model on a pyrochlore lattice interacting via the long-range RKKY interaction has been studied theoretically. ${ }^{25}$ According to this theory, LRO of localized moment with local $\langle 111\rangle$ Ising anisotropy is realized in the metallic state. In the present results, it should be noted that only a single phase transition due to the FM ordering is observed above $11 \mathrm{GPa}$; in particular, no additional phase transition is observed at $13 \mathrm{GPa}$ down to $100 \mathrm{mK}$. If the FM ordering originates from only the Ir moment, the ground-state entropy of the $\mathrm{Nd}$ moment is not relieved down to near $0 \mathrm{~K}$ in spite of a metallic state. Obviously, this scenario is contradictory to the aforementioned theoretical result. Therefore, it is highly possible that the FM ordering is mainly driven by the $\mathrm{Nd}$ moment although further measurements are required to elucidate the origin of the FM ordering. Furthermore, in general, the resistivity of localized $f$-electron systems decreases by a FM transition, because the $c-f$ exchange scattering is suppressed below the Curie temperature. ${ }^{26}$ The obtained results are consistent with this fact. Therefore, it is quite reasonable to say that the FM ordering originates from the Nd moment in the metallic state.

Next, we discuss the ordered state. According to the aforementioned theoretical study, in the case of an FM RKKY interaction, the theoretical result shows that the FM ordering of "two-in two-out" with the wave vector $\boldsymbol{q}=(0,0,0)$ or $(0,0,2 \pi / a)$ has been realized; this is an "ordered spin ice" state in a metallic state. ${ }^{25}$ Therefore, we propose that the pressure-induced transition of $\mathrm{Nd}_{2} \mathrm{Ir}_{2} \mathrm{O}_{7}$ at $3 \mathrm{~K}$ is the FM ordering of "two-in two-out" with $\boldsymbol{q}=(0,0,0)$ or $(0,0,2 \pi / a)$.

Figure 3 shows the pressure-temperature phase diagram obtained from the measurement of electrical resistance. $T_{\mathrm{MI}}$ is considered as the temperature at which $R(T)$ starts to increase abruptly, as shown in Fig. 1(a). $T_{\mathrm{O}}$ emerges suddenly at $10 \mathrm{GPa}$, which is close to the pressure at which the insulating phase

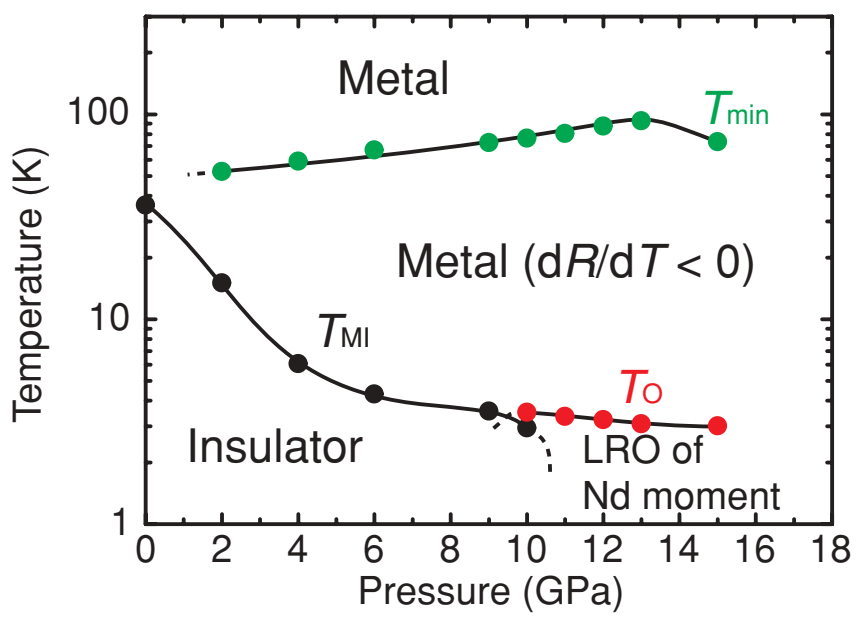

FIG. 3. (Color online) Phase diagram for $\mathrm{Nd}_{2} \mathrm{Ir}_{2} \mathrm{O}_{7}$. disappears. At pressures lower than $10 \mathrm{GPa}$, no resistance drop assigned to the magnetic LRO is observed in the plot of temperature dependence of resistance; therefore, there is no LRO of the $\mathrm{Nd}$ moment at least in the measured temperature region. Thus, the boundary pressure of the insulating phase should be around $11 \mathrm{GPa}$.

The MIT in $\mathrm{Nd}_{2} \mathrm{Ir}_{2} \mathrm{O}_{7}$ is monotonically suppressed by applying pressure. The pressure dependence of $T_{\mathrm{MI}}$ is negativeapproximately $-4 \mathrm{~K} / \mathrm{GPa}$ up to $6 \mathrm{GPa}$. This is similar to the values observed in other pyrochlore oxides, $\mathrm{Cd}_{2} \mathrm{Os}_{2} \mathrm{O}_{7}$ $(-4 \mathrm{~K} / \mathrm{GPa})^{12}$ and $\mathrm{Hg}_{2} \mathrm{Ru}_{2} \mathrm{O}_{7}(-5.4 \mathrm{~K} / \mathrm{GPa}) .{ }^{18}$ The application of pressure leads to structural deformation with a decrease in the Ir-O-Ir bond angle. However, we confirmed that this pressure effect is contradictory to the supposition made on the basis of the ionic radius dependence of $T_{\mathrm{MI}}$ in $L n_{2} \mathrm{Ir}_{2} \mathrm{O}_{7}$. Consequently, the result indicates that the decrease in the Ir-O-Ir bond length by the application of pressure strongly affects the electrical conductivity of $\mathrm{Ln}_{2} \mathrm{Ir}_{2} \mathrm{O}_{7}$.

From the phase diagram, we can say that the insulating phase below the MIT suppresses the emergence of the magnetic ordered phase. The pressure-induced FM ordered phase competes with the insulating phase. The MIT involves magnetic ordering with a weak FM component from $5 d$ electrons. At ambient pressure, the magnetic susceptibility measurement of a polycrystalline $\mathrm{Nd}_{2} \mathrm{Ir}_{2} \mathrm{O}_{7}$ sample provides no evidence for magnetic $\mathrm{LRO}$ of the $\mathrm{Nd}$ moment down to $2 \mathrm{~K}^{24}$ Because the internal field generated by a magnetic ordering due to $5 d$ electrons interacts with the $\mathrm{Nd}$ moments, the magnetic ordering of the $\mathrm{Nd}$ moment is considered to be suppressed or destroyed. The suppression of the MIT by the application of pressure leads to the magnetic ordering of the Nd moment via the RKKY interaction in the metallic state. Then, as $\mathrm{Nd}_{2} \mathrm{Ir}_{2} \mathrm{O}_{7}$ becomes more metallic due to pressure application, the RKKY interaction becomes stronger. Therefore, the appearance of the magnetic LRO phase at $3.5 \mathrm{~K}$ and $10 \mathrm{GPa}$ is probably due to the strengthening of the RKKY interaction by pressure application. Furthermore, because the magnetic LRO of the Nd moment at $3.5 \mathrm{~K}$ and $10 \mathrm{GPa}$ makes no opening of band gap, the MIT can occur below $T_{\mathrm{O}}$.

The localized Nd moments in the metallic phase interact with itinerant $5 d$ electrons via $c-f$ hybridization. We may consider that $\mathrm{Nd}_{2} \mathrm{Ir}_{2} \mathrm{O}_{7}$ in the metallic phase is a frustrated Kondo lattice system, as pointed out about $\operatorname{Pr}_{2} \mathrm{Ir}_{2} \mathrm{O}_{7}$ in Ref. 23. RKKY interaction stabilizes a magnetic LRO. On the other hand, the Kondo effect screens localized moments by a spin polarization cloud of conduction electrons. Consequently, a Fermi liquid state is formed by perfect screening of the magnetic moment. The magnetic phase diagram resulting from their competition is usually described by a model proposed by Doniach. ${ }^{27}$ The ground state depends on the strength of $c-f$ hybridization. $\mathrm{In} \mathrm{Nd}_{2} \mathrm{Ir}_{2} \mathrm{O}_{7}$, the application of pressure induces the magnetic LRO by the RKKY interaction. On the other hand, the pressure dependence of $T_{\mathrm{O}}$ was slightly negative at pressures higher than $11 \mathrm{GPa}$. As one possible explanation for the suppression of magnetic LRO, we may consider that the Kondo effect becomes dominant by the application of higher pressure. Then, a magnetic quantum critical point (QCP) is expected to appear at pressures greater than $15 \mathrm{GPa}$. Around the magnetic QCP, we have expected that the magnetic moment reduced by the Kondo effect strongly fluctuates on the 
frustrated pyrochlore lattice. A new physics on the frustrated pyrochlore lattice is underlying around QCP.

In summary, we have observed that the MIT in frustrated pyrochlore oxides $\mathrm{Nd}_{2} \mathrm{Ir}_{2} \mathrm{O}_{7}$ is suppressed by the application of pressure. Furthermore, we have found a new pressureinduced transition at pressures higher than $10 \mathrm{GPa}$. We have proposed that this transition is due to the FM ordering of the $\mathrm{Nd}$ moment, which is theoretically considered to be an "ordered spin ice" state in a metallic state. We have observed the phase competition between the MIT and the pressure-induced FM ordering. The pressure-induced magnetic LRO phase is suppressed slightly by applying further pressure but it remains at pressures of up to $15 \mathrm{GPa}$. Further studies are required in order to elucidate the details of the new magnetic LRO phase and to understand a physical property around magnetic QCP.

This work was supported by Grants-in-Aid for Scientific Research on Priority Areas "Novel States of Matter Induced by Frustration" (No. 19052005), Scientific Research on Innovation Areas "Heavy Electrons" (No. 21102518), and Scientific Research (S) (No. 19104009) and also by the Global COE Program, MEXT, Japan. *sakata@djebel.mp.es.osaka-u.ac.jp

${ }^{1}$ M. A. Subramanian, G. Aravamudan, and G. V. S. Rao, Prog. Solid State Chem. 15, 55 (1983).

${ }^{2}$ M. J. Harris, S. T. Bramwell, D. F. McMorrow, T. Zeiske, and K. W. Godfrey, Phys. Rev. Lett. 79, 2554 (1997).

${ }^{3}$ A. P. Ramirez, A. Hayashi, R. J. Cava, R. Siddharthan, and B. S. Shastry, Nature (London) 399, 333 (1999).

${ }^{4}$ J. S. Gardner, S. R. Dunsiger, B. D. Gaulin, M. J. P. Gingras, J. E. Greedan, R. F. Kiefl, M. D. Lumsden, W. A. MacFarlane, N. P. Raju, J. E. Sonier, I. Swainson, and Z. Tun, Phys. Rev. Lett. 82, 1012 (1999).

${ }^{5}$ M. Hanawa, Y. Muraoka, T. Tayama, T. Sakakibara, J. Yamaura, and Z. Hiroi, Phys. Rev. Lett. 87, 187001 (2001).

${ }^{6}$ S. Yonezawa, Y. Muraoka, Y. Matsushita, and Z. Hiroi, J. Phys. Condens. Matter 16, L9 (2001).

${ }^{7}$ S. Yonezawa, Y. Muraoka, Y. Matsushita, and Z. Hiroi, J. Phys. Soc. Jpn. 73, 819 (2004).

${ }^{8}$ S. Yonezawa, Y. Muraoka, and Z. Hiroi, J. Phys. Soc. Jpn. 73, 1655 (2004).

${ }^{9}$ Y. Taguchi, Y. Oohara, H. Yoshizawa, N. Nagaosa, and Y. Tokura, Science 291, 2573 (2001).

${ }^{10}$ Y. Machida, S. Nakatsuji, Y. Maeno, T. Tayama, T. Sakakibara, and S. Onoda, Phys. Rev. Lett. 98, 057203 (2007).

${ }^{11}$ A. W. Sleight, J. L. Gillson, J. F. Weiher, and W. Bindloss, Solid State Commun. 14, 357 (1974).

${ }^{12}$ D. Mandrus, J. R. Thompson, R. Gaal, L. Forro, J. C. Bryan, B. C. Chakoumakos, L. M. Woods, B. C. Sales, R. S. Fishman, and V. Keppens, Phys. Rev. B 63, 195104 (2001).

${ }^{13}$ H. S. Jarrett, A. W. Sleight, J. F. Weiher, J. L. Gillson, P. C. Frederick, G. A. Jones, R. S. Swingle, D. Swartzfager, J. E. Gulley, and P. C. Hoell, Valence Instability and Related Narrow-band Phenomena, edited by R. D. Parks (Plenum, New York, 1977), p. 545 .
${ }^{14}$ T. Takeda, M. Nagata, H. Kobayashi, R. Kanno, Y. Kawamoto, M. Takano, T. Kamiyama, F. Izumi, and A. W. Sleight, J. Solid State Chem. 140, 182 (1998).

${ }^{15}$ A. Yamamoto, P. A. Sharma, Y. Okamoto, A. Nakao, H. A. Katori, S. Niitaka, D. Hashizume, and H. Takagi, J. Phys. Soc. Jpn. 76, 043703 (2007).

${ }^{16}$ K. Matsuhira, Z. Hiroi, T. Tayama, S. Takagi, and T. Sakakibara, J. Phys. Condens. Matter 14, L559 (2002).

${ }^{17}$ I. Mirebeau, I. N. Goncharenko, P. Cadavez-Peres, S. T. Bramwell, M. J. P. Gingras, and J. S. Gardner, Nature (London) 420, 54 (2002).

${ }^{18}$ N. Takeshita, C. Terakura, Y. Tokura, A. Yamamoto, and H. Takagi, J. Phys. Soc. Jpn. 76, 063707 (2007).

${ }^{19}$ K. Matsuhira, M. Wakeshima, R. Nakanishi, T. Yamada, A. Nakamura, W. Kawano, S. Takagi, and Y. Hinatsu, J. Phys. Soc. Jpn. 76, 043706 (2007).

${ }^{20}$ D. Yanagishima and Y. Maeno, J. Phys. Soc. Jpn. 70, 2880 (2001).

${ }^{21}$ H. Fukazawa and Y. Maeno, J. Phys. Soc. Jpn. 71, 2578 (2002).

${ }^{22}$ J. N. Millican, R. T. Macaluso, S. Nakatsuji, Y. Machida, Y. Maeno, and J. Y. Chan, Mater. Res. Bull. 42, 928 (2007).

${ }^{23}$ S. Nakatsuji, Y. Machida, Y. Maeno, T. Tayama, T. Sakakibara, J. van Duijn, L. Balicas, J. N. Millican, R. T. Macaluso, and J. Y. Chan, Phys. Rev. Lett. 96, 087204 (2006).

${ }^{24} \mathrm{~K}$. Matsuhira, abstract [27prk-5] in 2009 autumn meeting of the Physical Society of Japan, Sept. 25-28, 2009; submitted to J. Phys. Soc. Jpn.

${ }^{25}$ A. Ikeda and H. Kawamura, J. Phys. Soc. Jpn. 77, 073707 (2008).

${ }^{26}$ T. Kasuya, Prog. Theor. Phys. 16, 99 (1956).

${ }^{27}$ S. Doniach, Physica B 91, 231 (1977). 